\title{
Influence of the Micromechanics Models and Volume Fraction Distribution on the Overall Behavior of SiC/Al Functionally Graded Pressurized Cylinders
}

\author{
Marcelo Silva Medeiros Jr. ${ }^{a *}$ (iD \\ Evandro Parente Jr. ${ }^{\mathrm{a}}$ (D) \\ Antônio Macário Cartaxo de Melo ${ }^{a}$ \\ a Departamento de Engenharia Estrutural e Construção Civil, Centro de Tecnologia, Universidade Federal do Ceará, Fortaleza, CE, Brasil. \\ E-mail: marcelomedeiros@ufc.br_evandro@ufc.br, macario@ufc.br \\ * Corresponding Author
}

http://dx.doi.org/10.1590/1679-78255433

\begin{abstract}
The assessment of the differences in results obtained from various micromechanics homogenization schemes, as well as the implications of assuming different volume fraction profiles was carried out in the present work. The functionally graded composite chosen for the analysis was Al-SiC and comparisons were made in terms of stress and strain distributions along the wall of an internally pressurized hollow cylinder. Different micromechanics homogenization schemes were implemented into Abaqus as user-defined subroutines (UMAT). The numerical simulations were compared to a set of analytical solutions available on the literature. The obtained results varied substantially according to the homogenization scheme employed. It was also found that the type of function chosen to describe the volume fraction distribution plays a major role on the development of the hoop stresses. Additionally, the finite element analysis showed significant stress variation when the actual volume fraction distribution was used. These gradients did not appear when the same profile was approximated by smooth exponential functions. This paper points out some important issues related to common practices associated with the analysis of FGM composites and serves as an overture to a more in-depth discussion of such problems.
\end{abstract}

\section{Keywords}

Micromechanics, FGM, Metal Matrix Composite.

\section{INTRODUCTION}

Metal Matrix Composites (MMCs) reinforced with ceramic particulates combine the ductility and toughness of metals with the high strength, high stiffness and low thermal conductivity of the ceramics. This combination of qualities leads to composites with a superior thermo-mechanical performance. The use of MMCs as a major component in aerospace, automotive, maritime and petroleum industries has been established over the recent years. This was the result of the widespread availability of relatively inexpensive reinforcements, especially particulates in the micro and nano scales. Additionally, the development of various processing and manufacturing technologies resulted in reproducible microstructures and final products with more compliant properties.

Reduction in structural weight can be achieved, not only by reducing the alloy density, but also by increasing its stiffness and strength. For example, an increase in modulus, attained by substituting a wrought aluminum part by a particulate reinforced aluminum alloy, can result in overall weight reduction (Kainer 2006). Reinforcement may be in the form of continuous fibers, chopped fibers, whiskers, platelets or particulates. Common reinforcement particulates 
include, but are not restricted to, carbides (e.g., $\mathrm{SiC}, \mathrm{B}_{4} \mathrm{C}$ ), nitrides (e.g., $\mathrm{Si}_{3} \mathrm{~N}_{4}, \mathrm{AlN}$ ), oxides (e.g., $\mathrm{Al}_{2} \mathrm{O}_{3}, \mathrm{SiO}_{2}$ ), as well as elemental materials (e.g., $C, S i)$. Silicon Carbide $\left({ }_{S i C}\right)$, for example, is a particulate powder that is commonly used in aluminum and magnesium MMCs.

The demand for a high-performance thermal barrier to withstand the elevated temperatures from space vehicles re-entries, originated a new class of composites called functionally graded materials (FGMs) (Koizumi 1997). Functionally graded materials are essentially a class of advanced composites formed by two or more constituent phases (matrix and inclusions) with a gradual and continuously varying microstructural composition. This leads to structural elements with different functional performance within different sections of the same part. The technologies to produce FGM composites have not reached their maturity yet and are expected to have an ever-growing impact on the design and development of new components and structures in the years to come.

There are currently several different approaches to successfully introduce ceramic particulates into an alloy melt. Processes such as, injection of powders entrained in an inert gas, addition of particles by mechanical agitation, pushing of the particulates in the melt by using reciprocating rods, dispersion of the particulates by ultrasound are commonly used in the industry (Bains, Sidhu, and Payal 2016). Nonetheless, the dispersion of the particulates in the melt using centrifugal acceleration is the method of choice when producing metal pipes, accounting for $15 \%$ of the total casting output of the world in terms of tonnage (Stefanescu 2009).

Most of the references on the literature of FGMs employ, indiscriminately, the rule of mixture or Mori-Tanaka schemes to obtain the effective material properties. This is done regardless of the type, shape, form or size of the composite constituents (Shen and Wang 2012; Akbarzadeh, Abedini, and Chen 2015). However, the complexity of the problem at hand requires more attention when choosing the micromechanical homogenization approach. In order to shed some light on this issue the work presented here was structured in a way that allowed quantifying the discrepancies among four different homogenization schemes as well as different ways to parameterize the volume fraction distribution.

The paper was divided into two main parts. Firstly, a set of user-defined material subroutines (UMAT) were developed to serve as the framework for the Finite Element Analysis. These codes were validated against analytical solutions available for an internally pressurized, thick-wall FGM cylinder. Secondly, a parametric study involving different volume fraction distributions and different homogenization schemes was carried out and discussed. The impact of these variables was assessed in terms of radial and hoop stresses/strain profiles along the cylinder wall.

\section{THEORETICAL FRAMEWORKS}

In the process of micromechanical homogenization, the macroscopic equivalent parameters are obtained through the structural responses of representative volume elements (RVEs) which are defined on a finer scale. In this type of homogenization approach, a material point on the macroscopic level can be related to a representative volume element on the microscopic level where stresses and strains prevail as varying micro-fields, assuming a statistically homogeneous distribution of the heterogeneities (inclusions) throughout the material (Vignoli and Savi 2018). The macro-stresses and macro-strains which characterize the mechanical state of the macroscopic material point can be treated as volumetric averages of the microscopic fields over a certain volume (Vignoli and Savi 2018). These volumes must be chosen large enough to contain a certain number of inclusions, but small when compared to any length scale over which the average loading or deformation of the composite varies.

In the particular case of FGMs the distribution of heterogeneities presents a spatial variation such that the condition of a statistically homogeneous microstructure is not observed and the use of effective properties must be understood simply as a reasonable estimate of the macro properties (Gross and Seelig 2011). The Mean Field Theory is a well-known approach to this problem, and which is built upon the concept of an imaginary RVE. It accounts for the interaction between subdomains pertinent to the various phases and finds the new averages that satisfy the macroscopic boundary conditions (Perdahcıoğlu and Geijselaers 2011). As is typical in mean field micromechanics models, fourth-order concentration tensors relate the average stress or average strain tensors in inclusions and matrix to the average macroscopic stress or strain tensor, respectively.

\subsection{Mean-Field Homogenization}

Macroscopic homogeneity is defined by postulating that strain and stress averages, for homogeneous boundary conditions, are the same over randomly chosen RVE. This is then equivalent to postulation of space independence of ensemble strain and stress averages. The averaged quantities are straightforwardly the integral over a specified region divided by its volume. More specifically, if the position vector is denoted by $x$, the volume-averaged stresses and strains, 
represented here by the brackets \langle\rangle , are defined as the average of the pointwise stress or strain over the volume $V$ as in the following expressions (Hashin 1963):

$$
\begin{aligned}
& \left\langle\sigma_{i j}\right\rangle=\frac{1}{V} \int_{V} \sigma_{i j}(x) d V \\
& \left\langle\varepsilon_{i j}\right\rangle=\frac{1}{V} \int_{V} \varepsilon_{i j}(x) d V
\end{aligned}
$$

Consider a two-phase composite consisting of an isotropic elastic matrix that occupies a volume $V_{M}$ and an inclusion phase that occupies a volume $V_{\text {I }}$ where the sum of these two volumes corresponds to the total domain $V$. Consider also that these two phases are perfectly bonded at their interfaces forming a continuum body. The corresponding volume fractions of matrix and inclusions are given by $v_{M}=V_{M} / V$ and $v_{I}=V_{I} / V$, from where it follows that $v_{M}+v_{I}=1$ and $v_{M}=1-v_{I}$. The relationships between each phase average and the overall average can be established in terms of the preceding definitions as:

$$
\begin{aligned}
& \left\langle\sigma_{i j}\right\rangle=v_{I}\left\langle\sigma_{i j}^{I}\right\rangle+\left(1-v_{I}\right)\left\langle\sigma_{i j}^{M}\right\rangle \\
& \left\langle\varepsilon_{i j}\right\rangle=v_{I}\left\langle\varepsilon_{i j}^{I}\right\rangle+\left(1-v_{I}\right)\left\langle\varepsilon_{i j}^{M}\right\rangle
\end{aligned}
$$

The average inclusion and matrix micro-fields (stress or strain) over the respective domains $V_{l}$ and $V_{M}$ are given by:

$$
\begin{aligned}
& \left\langle\sigma_{i j}^{I}\right\rangle=\frac{1}{V_{I}} \int_{V_{I}} \sigma_{i j}(x) d V ; \quad\left\langle\sigma_{i j}^{M}\right\rangle=\frac{1}{V_{M}} \int_{V_{M}} \sigma_{i j}(x) d V \\
& \left\langle\varepsilon_{i j}^{I}\right\rangle=\frac{1}{V_{I}} \int_{V_{I}} \varepsilon_{i j}(x) d V ; \quad\left\langle\varepsilon_{i j}^{M}\right\rangle=\frac{1}{V_{M}} \int_{V_{M}} \varepsilon_{i j}(x) d V
\end{aligned}
$$

In regards with the volume-averaged stress, it can be said that, in the absence of body forces the equilibrium conditions $\sigma_{i k, k}=0$ and $x_{j, k}=\delta_{j k}$ imply that the derivative of the product of the two is expressed by:

$$
\left(\sigma_{i k} x_{j}\right)_{, k}=\sigma_{i k, k} x_{j}+\sigma_{i k} x_{j, k}=\left(\sigma_{i k} x_{j}\right)_{, k}=\sigma_{i k} \delta_{j k}=\sigma_{i j}
$$

Inserting Equation (7) into Equation (1) leads to the representation of the macro stresses.

$$
\left\langle\sigma_{i j}\right\rangle=\frac{1}{V} \int_{V}\left(\sigma_{i k} x_{j}\right),{ }_{k} d V
$$

The Gauss' divergence theorem can be used to express the average stress by an integral over the boundary $\partial \mathrm{V}$ of the averaging domain $V$. It is assumed that the microscopic fields are differentiable throughout the entire domain, which does not hold true for heterogeneous materials with discontinuously varying properties. Now consider that the volume $V$ consists of two subdomains $V_{1}$ and $V_{2}$ with different properties according to Figure 1 . Consider also the interfacial surface between the two volumes as the boundary $S$. 


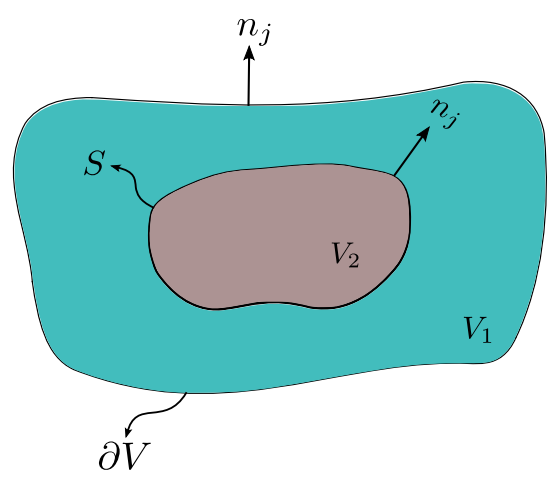

Figure 1: Volume $V$ with an internal interface $S$.

It can be seen that across the interface $S$, the stresses and displacements may not be differentiable. This challenge can be overcome if the divergence theorem is applied separately to the two subdomains where $S$ appears, once as the boundary of $V_{2}$ (with unit outward normal vector $n_{j}$ ) and also as the inner boundary of $V_{1}$ (with unit outward normal vector $\left.-n_{j}\right)$. Considering the corresponding traction vectors of $V_{1}$ and $V_{2}$ as $t_{i}^{(1)}$ and $t_{i}^{(2)}$, respectively we have:

$V\left\langle\sigma_{i j}\right\rangle=\int_{V_{1}} \sigma_{i j} d V+\int_{V_{2}} \sigma_{i j} d V=\int_{\partial V} t_{i} x_{j} d A+\int_{S}\left(t_{i}^{(2)}+t_{i}^{(1)}\right) x_{j} d A$

However the equilibrium condition implies that $\boldsymbol{t}_{i}^{(1)}=-\boldsymbol{t}_{\boldsymbol{i}}^{(2)}$. As a result, for quasi-static problems, in the absence of body forces, the average stress in a given RVE is prescribed only in terms of the boundary traction:

$\left\langle\sigma_{i j}\right\rangle=\frac{1}{V} \int_{\partial V} t_{i} x_{j} d A$

A similar expression for strain can also be developed if the strain tensor $\varepsilon_{i j}$ is substituted by the corresponding displacement vector $\boldsymbol{u}$ into the definition of macrostrains (Equation 2). If the domain $\mathrm{V}$ is subjected to a surface displacement $\boldsymbol{u}(\boldsymbol{x})$ and there is a perfect contact between the phases $\left(\boldsymbol{u}_{i}^{(1)}=\boldsymbol{u}_{i}^{(2)}\right)$, the divergence theorem can be applied in a similar manner, yielding:

$$
\begin{aligned}
& 2 V\left\langle\varepsilon_{i j}\right\rangle=\int_{V}\left(u_{i, j}+u_{j, i}\right) d V=\int_{\partial V}\left(u_{i} n_{j}+u_{j} n_{i}\right) d A+\int_{S}\left[\left(u_{i}^{(1)}-u_{i}^{(2)}\right) n_{j}+\left(u_{j,}^{(1)}-u_{j,}^{(2)}\right) n_{i}\right] d S \\
& \left\langle\varepsilon_{i j}\right\rangle=\frac{1}{2 V} \int_{\partial V}\left(u_{i} n_{j}+u_{j} n_{i}\right) d A
\end{aligned}
$$

Equations (10) and (11) are called average stress and average strain theorems (Hill 1964).

\subsection{Homogenization Methods}

The basic premise of the mean-field homogenization methods is that the volume-averaged strains and stresses can be related to one another or to the overall boundary conditions by means of some fourth-order concentration tensors. Therefore, the main objective is to define a suitable stress or strain concentration tensor that relates the average stress or strain within the inclusion to the remotely applied boundary conditions. In this work all the formulations are presented in terms of strain concentration tensors, nonetheless similar expressions can be found if stress concentration tensors are used instead. The fourth-order strain concentration tensors that correlate the phase-averaged strains within the inclusion to the overall macrostrains and the averaged strains over the matrix are given respectively as follows:

$$
\begin{aligned}
& \left\langle\varepsilon_{i j}^{I}\right\rangle=A_{i j k l}\left\langle\varepsilon_{k l}\right\rangle \\
& \left\langle\varepsilon_{i j}^{I}\right\rangle=B_{i j k l}\left\langle\varepsilon_{k l}^{M}\right\rangle
\end{aligned}
$$


Different homogenization schemes will differ in the way the tensors $A_{i j k l}$ and $B_{i j k l}$ are defined, but in all of them the averaged macro-stiffness tensor of the two-phase composite is written in terms of one of the two following expressions:

$$
\begin{aligned}
& \left\langle C_{i j k l}\right\rangle=\left[v_{I} C_{i j m n}^{I} B_{m n o p}+\left(1-v_{I}\right) C_{i j o p}^{M}\right]\left[v_{I} B_{o p k l}+\left(1-v_{I}\right) I_{o p k l}\right]^{-1} \\
& \left\langle C_{i j k l}\right\rangle=C_{i j k l}^{M}+v_{I}\left(C_{i j m n}^{I}-C_{i j m n}^{M}\right) A_{m n k l}
\end{aligned}
$$

where the fourth-rank $C_{i j k l}^{I}$ and $C_{i j k l}^{M}$ tensors are the inclusion and matrix stiffness, respectively. Mean field homogenization schemes are relatively simple to implement within the framework of finite element codes and are very efficient from a computational standpoint. The homogenization schemes used in this work were implemented as Abaqus custom user subroutines to define the constitutive behavior of the material (UMATs).

\subsection{Eshelby's Tensor}

J. D. Eshelby developed a very important micromechanics solution for a specific class of inclusions inside an unbounded domain (Eshelby 1957). The inclusions are defined within an infinite homogeneous, isotropic, and elastic medium (matrix) undergoing a change in shape and size. He used the concept of eigenstrains ( $\left.\varepsilon_{k l}^{*}\right)$ to prove that under the constraint of the matrix, the inclusion has an arbitrary homogeneous strain and stress fields. He proposed that the stress concentrated in an inhomogeneity can be equally represented as the stress concentrated by an inclusion undergoing an arbitrary type of transformation strain produced without external forces (eigenstrain). In this problem, the equilibrium strain and stress are to be determined in the case where a strain is prescribed in a certain volume within an infinitely large homogeneous material.

If the eigenstrains inside the elliptical inclusion are constant, then the total strain inside the inclusion are constant as well. Therefore, the average strain inside the ellipsoidal inclusion $\left\langle\epsilon_{i j}^{I}\right\rangle$, is linearly related to the eigenstrains by the fourth order Eshelby's tensor $\mathbb{S}_{i j k l}$ :

$$
\left\langle\epsilon_{i j}^{I}\right\rangle=\mathbb{S}_{i j k l}\left\langle\epsilon_{k l}^{*}\right\rangle
$$

This tensor presents the minor symmetry, but in general lacks the major symmetry:

$$
\mathbb{S}_{i j k l}=\mathbb{S}_{j i k l}=\mathbb{S}_{i j l k} \neq \mathbb{S}_{k l i j}
$$

The concepts of eigenstrain and the ensuing Eshelby's tensor, that correlates it to the average strains inside the inclusion allows for a simpler way to tackle the homogenization problems. By defining difference fields and defining the strain field inside the inclusions as an equivalent eigenstrain field a formal definition for the strain concentration factor $A_{i j k l}$ can be found. The geometry of an ellipsoidal inclusion with a semi axis $a_{i}$ is given by

$$
\frac{x_{1}^{2}}{a_{1}^{2}}+\frac{x_{2}^{2}}{a_{2}^{2}}+\frac{x_{3}^{2}}{a_{3}^{2}} \leq 1
$$

Assuming $a_{1}>a_{2}>a_{3}$, the components of the Eshelby's tensor are defined as:

$$
\mathbb{S}_{1111}=\frac{3}{8 \pi(1-v)} a_{1}^{2} I_{11}+\frac{1-2 v}{8 \pi(1-v)} I_{1}
$$


$\mathbb{S}_{1133}=\frac{1}{8 \pi(1-v)} a_{3}^{2} I_{13}-\frac{1-2 v}{8 \pi(1-v)} I_{1}$

$\mathbb{S}_{1212}=\frac{a_{1}^{2}+a_{2}^{2}}{16 \pi(1-v)} I_{12}+\frac{1-2 v}{16 \pi(1-v)}\left(I_{1}+I_{2}\right)$

All other non-zero components are obtained by cyclic permutation of $(1,2,3)$. The components which cannot be obtained this way are zero (Mura 1982); for instance $\mathbb{S}_{1112}=\mathbb{S}_{1223}=\mathbb{S}_{1232}=0$. The terms $l_{i}$ can be expressed by standard elliptic integrals of the form:

$I_{1}=\frac{4 \pi a_{1} a_{2} a_{3}}{\left(a_{1}^{2}-a_{2}^{2}\right) \sqrt{a_{1}^{2}-a_{3}^{2}}}\{F(\theta, k)-E(\theta, k)\}$

$I_{3}=\frac{4 \pi a_{1} a_{2} a_{3}}{\left(a_{2}^{2}-a_{3}^{2}\right) \sqrt{a_{1}^{2}-a_{3}^{2}}}\left\{\frac{a_{2} \sqrt{a_{1}^{2}-a_{3}^{2}}}{a_{1} a_{3}}-E(\theta, k)\right\}$

where:

$F(\theta, k)=\int_{0}^{\theta} \frac{1}{\sqrt{1-k^{2} \sin ^{2}(w)}} d w ; \quad E(\theta, k)=\int_{0}^{\theta} \sqrt{1-k^{2} \sin ^{2}(w)} d w$

$\theta=\sqrt{\arcsin \left(1-a_{3}^{2} / a_{1}^{2}\right)} ; \quad k=\sqrt{\left(a_{1}^{2}-a_{2}^{2}\right) /\left(a_{1}^{2}-a_{3}^{2}\right)}$

The remaining components of $l_{i}$ are obtained by the following inter-relationships:

$I_{1}+I_{2}+I_{3}=4 \pi$

$3 I_{11}+I_{12}+I_{13}=4 \pi / a_{1}^{2}$

$3 a_{1}^{2} I_{11}+a_{2}^{2} I_{12}+a_{3}^{2} I_{13}=3 I_{1}$

$I_{12}\left(a_{1}^{2}-a_{2}^{2}\right)=\left(I_{2}-I_{1}\right)$

In this work, the SiC particles were approximated by spheres $\left(a_{1}=a_{2}=a_{3}\right)$ therefore the resulting Eshelby's tensor is given by:

$\mathbb{S}_{1111}=\mathbb{S}_{2222}=\mathbb{S}_{3333}=\frac{7-5 v}{15(1-v)}$ 


$$
\begin{aligned}
& \mathbb{S}_{1122}=\mathbb{S}_{2233}=\mathbb{S}_{3311}=\mathbb{S}_{1133}=\mathbb{S}_{2211}=\mathbb{S}_{3322}=\frac{5 v-1}{15(1-v)} \\
& \mathbb{S}_{1212}=\mathbb{S}_{2323}=\mathbb{S}_{1313}=\frac{4-5 v}{15(1-v)}
\end{aligned}
$$

\section{All other components are zero.}

\subsection{Voigt and Reuss Models}

The two simplest homogenization schemes are the Voigt (Voigt 1889) and Reuss (Reuss 1929) models. The Voigt model is also known as Rule of Mixtures (RoM) and assumes a uniform strain field within the RVE, which means that both matrix and inclusions are subjected to the same strain, $\left\langle\epsilon_{i j}^{I}\right\rangle=\left\langle\epsilon_{i j}^{M}\right\rangle$. It follows from this assumption that the strain concentration factor equals the unit tensor, yielding the following expression when substituted in Equation (14):

$$
B_{i j k l}^{\text {Voigt }}=I_{i j k l} \rightarrow C_{i j k l}^{\text {Voigt }}=v_{I} C_{i j k l}^{I}+\left(1-v_{I}\right) C_{i j k l}^{M}
$$

This model can also be understood as a linearly weighted average of the properties of each phase. The Reuss model, on the other hand, assumes a uniform stress inside the RVE, meaning that all phases experience the same stress. This premise leads to a strain concentration factor that corresponds to the ratio between the stiffness of the matrix over the stiffness of the inclusion. Substituting the resulting strain concentration factor on Equation (14) and knowing that $C_{i j k l}^{-1}=S_{i j k l}$ one obtains:

$$
B_{i j k l}^{\mathrm{Re} u s s}=\left(C_{i j m n}^{I}\right)^{-1} C_{m n k l}^{M} \rightarrow S_{i j k l}^{\text {Reuss }}=v_{I} S_{i j k l}^{I}+\left(1-v_{I}\right) S_{i j k l}^{M}
$$

These two models yield results too far apart from each other and do not take into account the shape or the orientation of the inclusions. Moreover, the interaction between inclusions inside the matrix is not accounted for, which renders the two schemes useless for medium to high concentration of inclusions. However, the two models serve the purpose of setting the extreme (upper and lower) bounds which no other model can exceed.

\subsection{Mori-Tanaka Model}

Except for Reuss and Voigt models, all the other mean field homogenization schemes are based on the fundamental work of Eshelby, with the Mori-Tanaka model being one of the most prominent models in this category. In their original paper, Mori and Tanaka (1973) treated the problem of homogeneous inclusions by considering that in a dilute distribution of non-interacting inclusions the constant stress or strain field of the external load prevails. For that matter the authors used the concept of equivalent eigenstrains.

The Mori-Tanaka homogenization scheme considers the strain concentration tensor to be equal to that of a single inclusion problem, which in turn means that the inclusions in the RVE experience a far-field strain equal to the average strain in the matrix. Benveniste (1987) reformulated the work of Mori and Tanaka using a direct approach to define and compute the effective homogenized moduli. The strain concentration tensor in Mori-Tanaka's model is given by:

$$
A_{i j k l}^{M T}=\left[I_{i j k l}+v_{f} \mathbb{S}_{i j m n} S_{m n o p}^{M}\left(C_{o p k l}^{I}-C_{o p k l}^{M}\right)\right]^{-1}
$$

The Homogenized Modulus is obtained by a direct substitution of Equation (29) into Equation (15).

\subsection{Self-Consistent Model}

The Self-Consistent homogenization scheme has been originally developed to estimate the equivalent stiffness of polycristals and consider the interaction of the matrix and the grains following Eshelby's formulation. This approach was originally proposed by Hill $(1964 ; 1965)$ and focused on spherical particles and continuous aligned fibers. The Self- 
Consistent approach to homogenization is based upon the idea that the existence of a single in-homogeneity does not alter the effective material properties in a system with many inclusions. Therefore, the inclusions interact with each other through the effective medium, which means that the localization tensors will be based on the effective medium properties rather than the matrix material properties (Christensen 1990). Conversely, the local interaction of the inclusions with the surrounding matrix material remains unaltered.

Self-Consistent models are also referred to as embedding models. This is so because the composite properties are assumed to be the same as the averaged properties of a single particle embedded into an infinite matrix with a given stiffness $C_{i j k l}^{\text {trial }}$ yet to be found. The trial matrix properties are initially unknown; however, numerical solutions are easily obtained by an iterative algorithm (Chou, Nomura, and Taya 1980). The Self-Consistent strain concentration tensor is given by:

$$
B_{i j k l}^{S C}=\left(I_{i j k l}+\mathbb{S}_{i j m n} S_{m n o p}^{\text {trial }}\left[C_{o p k l}^{I}-C_{o p k l}^{\text {trial }}\right]\right)^{-1}
$$

However, the Self-Consistent method is known to overestimate the influence of the interaction among the inclusions, especially for softer matrices (Benveniste 2008). Christensen and Lo (1979) proposed an improved methodology to address this issue and make it yield the correct asymptotic behavior for rigid inclusions as its volume fraction approaches $100 \%$. This scheme was named Generalized Self-Consistent Method and considers that the particulate phase is first surrounded by some matrix material and then embedded in the effective medium. As the Generalized Self-Consistent Method is widely regarded as superior when compared to the original approach (Tucker III and Liang 1999), it was adopted in this study. The detailed formulation of the model in terms of effective bulk and shear moduli is given in Christensen and Lo (1979) and Christensen (1990) and are not repeated here for sake of brevity.

\section{METHODOLOGY}

In order to conduct the Finite Element Analysis of the FGM pressurized pipes in Abaqus, a user material subroutine (UMAT) was developed where the various homogenization schemes were implemented. The detailed description of the development and validation of the tasks conducted in this work is described as follows.

\subsection{Subroutines Development and Validation}

The first step towards this goal was to make sure that the stresses and strains obtained by the UMAT, given a preestablished modulus profile, were in accordance with the analytical solutions of this of problem. This was done prior to the implementation of the actual homogenization schemes and relied upon an already homogenized radial variation of the elastic modulus $(E)$ along the pipe wall. At this stage the UMAT would get the three Cartesian coordinates of each Gauss (integration) point passed on by Abaqus and transform it to cylindrical coordinates. The homogenized modulus, at each Gauss point, was then obtained by the designated equation for the elastic modulus as a function of radial position. Finally, the three-dimensional constitutive matrices were assembled, and the stress vector calculated.

A set of analytical solutions found in the literature was used in this work to verify the accuracy of the numerical results. The analytical expressions for isothermal radial and hoop stresses were given by Li and Peng (2009) for an FGM cylinder with an inner radius $a$, and outer radius $b$, subjected to an internal pressure $p_{i}$ and no external pressure. The authors assumed a fixed Poisson's ratio, $v(r)=v$ and established that the elastic modulus varied radially according to a power law given:

$$
E(r)=E_{0}\left(\frac{r}{b}\right)^{\beta}
$$

Where $E_{0}$ is the modulus at the outer surface of the pipe and $B$ is a curve shape parameter. The corresponding expressions for radial and hoop stresses are

$$
\sigma_{r}(r)=-p\left(\frac{a}{r}\right)^{(2+k-\beta) / 2}\left[\frac{b^{k}-r^{k}}{b^{k}-a^{k}}\right]
$$


$\sigma_{\theta}(r)=p\left(\frac{a}{r}\right)^{(2+k-\beta) / 2} \frac{1}{b^{k}-a^{k}}\left[\frac{2-(\beta+k) v}{\beta+k-2 v} b^{k}-\frac{2-(\beta-k) v}{\beta-k-2 v} r^{k}\right]$

$k=\sqrt{\beta^{2}+4-4 \beta v}$

The finite element model consisted of $1 / 4$ of a cylindrical section subjected to an internal pressure and with a traction-free outer surface. It had $200 \mathrm{~mm}$ in height and the inner and outer radius were, $a=100$ and $b=150 \mathrm{~mm}$. There were 15 elements along the wall thickness and the element employed was the 20 node quadratic element (C3D20). An internal pressure of $10 \mathrm{MPa}$ was applied at the inner surface of the cylinder, while no boundary conditions were imposed on the outer surface. The model and the radial stress field can be seen in Figure 2.

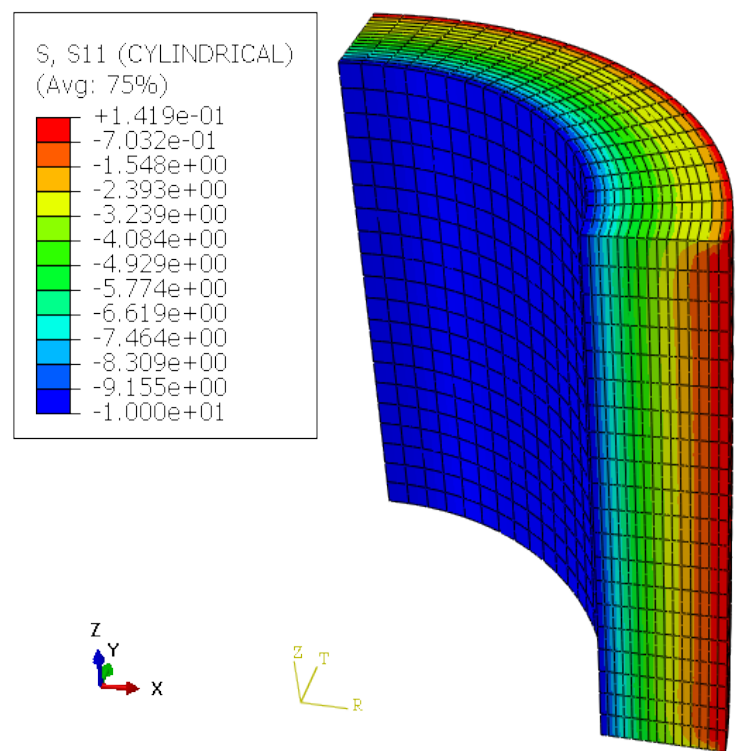

Figure 2: Radial Stress Field from Finite Element Analysis.

The numerical results for three different values of the exponent $\beta$ in Equation (31) were compared with results from Equations (32) and (33). The normalized radial and hoop stress profiles are given in Figure 3 . As can be seen, the results were deemed satisfactory in spite of minor differences on the hoop stresses. This is believed to be caused by the use of 3D FE model while the analytical solution is based on a plane strain configuration.

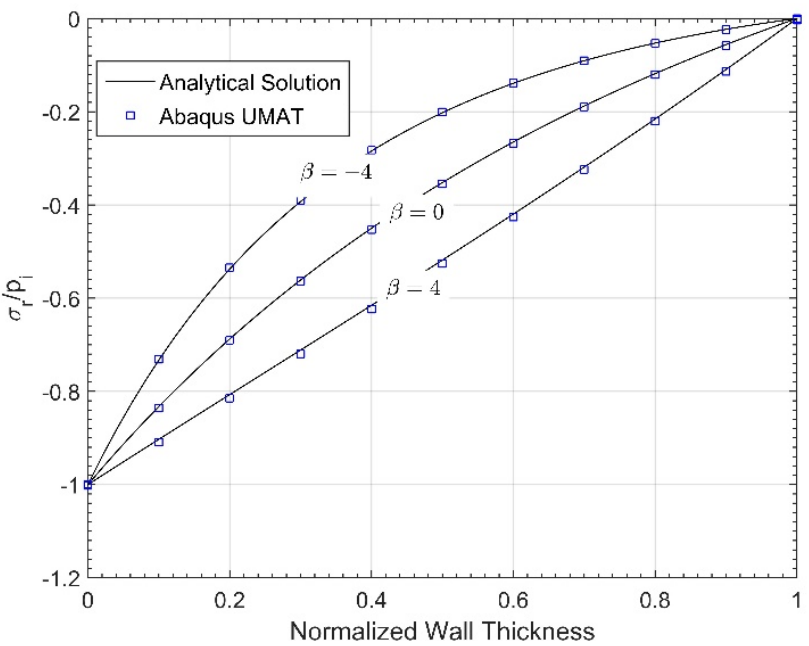

(a) Radial stress profiles

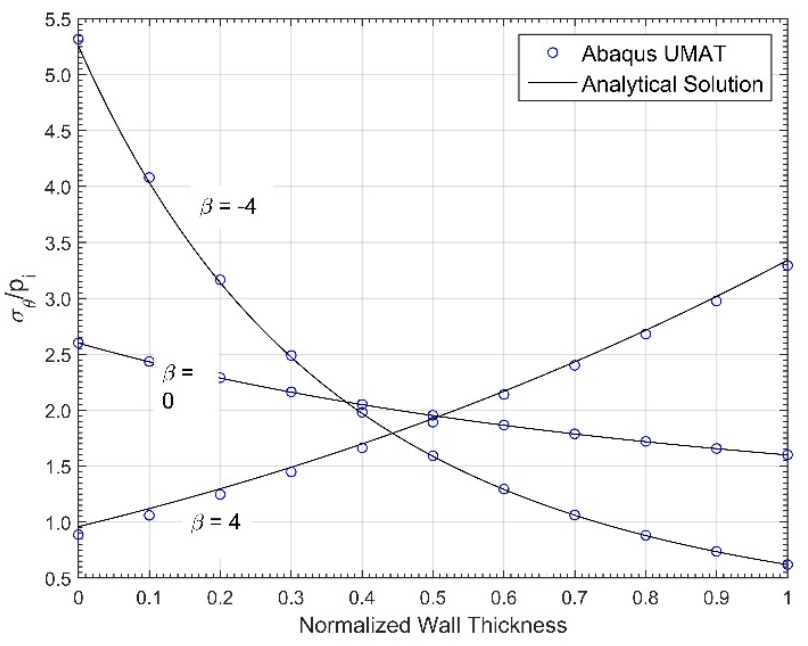

(b) Hoop stress profiles

Figure 3: Stress profiles along the wall thickness 


\subsection{Implementation of the Homogenization Schemes}

The next step on the development of the UMATs was to implement the various homogenization schemes discussed in this study. In order to establish a baseline for comparison among all models, several experimental studies on Al/SiC MMCs (Premkumar et al., 1992; Liaw et al. 1995; El-Eskandarany 1998; Santhosh Kumar et al. 2009; Upadhyay and Singh 2012; Qu, Lou, and Li 2016) along with data from various Al/SiC MMCs manufacturers' datasheets (MATWEB 2018), were compiled and presented in Figure 4 . The studies encompassed various volume fractions of SiC ranging from $0 \%$ to a little over $70 \%$. It is worth noting that these studies used different aluminum alloys and SiC particles with various gradations.

The four homogenization schemes in discussion here were firstly implemented as MATLAB standalone programs in order to check for any errors prior to the implementation as subroutines within the UMAT. This effort also served the purpose of showing which model ranked the best amongst the four, over various volume fraction ranges (low medium and high). This is important because the effective measurement of the elastic modulus and Poisson's ratio along the thickness of an FGM pipe is very difficult to achieve, whereas the measurement of the volume fraction distribution along the same thickness is more amenable from an experimental standpoint.

The elastic modulus and the Poisson's ratio presented in Figure 4 were calculated from the homogenized compliance tensor of each model according to the following expressions:

$$
E=\frac{1}{S_{1111}} ; \quad v=-\frac{S_{1122}}{S_{1111}}
$$

The results obtained by homogenization methods addressed in this work are presented in Figure 4 . These results show that, despite its enormous popularity, the use of the Voigt model (rule of mixtures) to predict the homogenized modulus of $\mathrm{Al} / \mathrm{SiC}$ MMCs must be avoided due to the accentuated differences from the experimental results. From this dataset, it can also be seen that the Generalized Self-Consistent and the Mori-Tanaka methods provided compatible results. The Generalized Self-Consistent method showed better results for elastic modulus estimation, especially for volume fractions above 30\%. Conversely the Mori-Tanaka model yielded Poisson's ratios closer to the actual measured values. After this initial assessment, the four homogenization schemes were then coded as UMATs, one for each model and used in the parametric study.

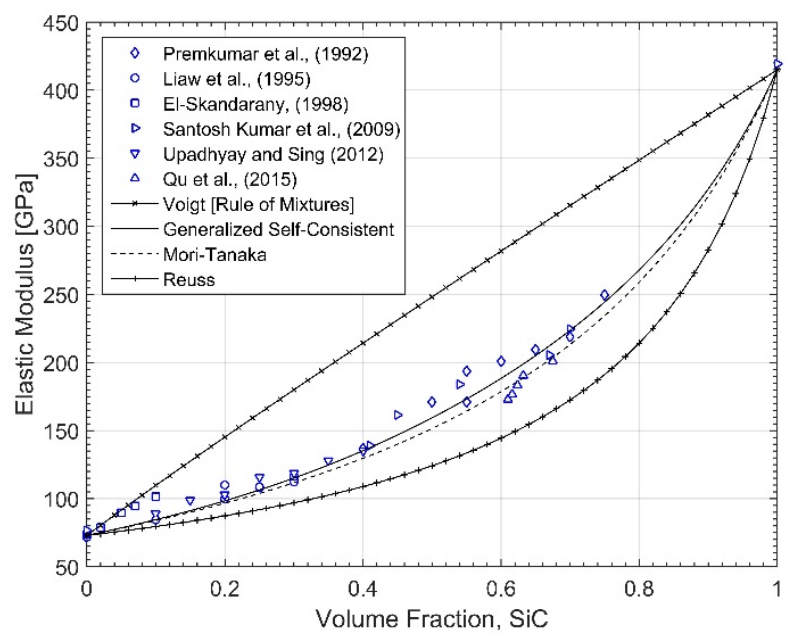

(a) Elastic Modulus per volume fraction

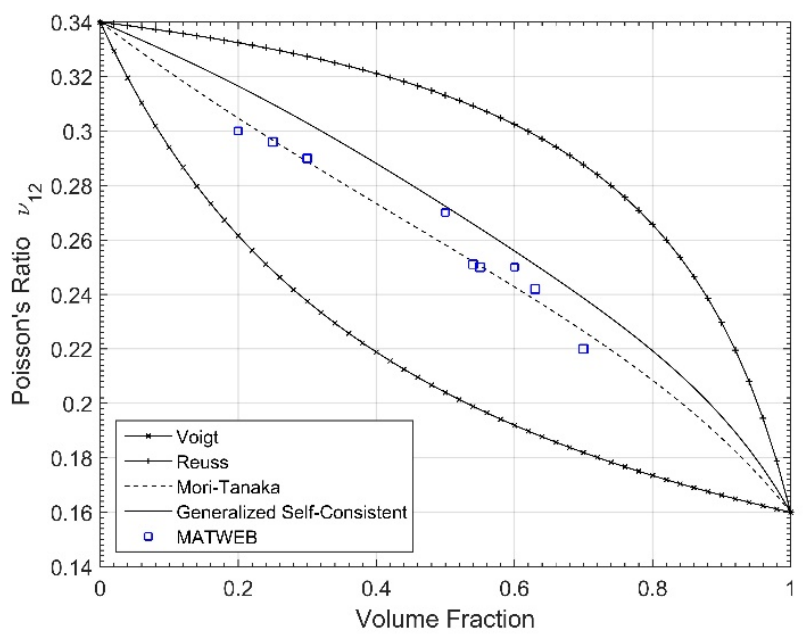

(b) Poisson's Ratio per volume fraction

Figure 4: Homogenization Schemes versus Experimental Data

\subsection{Parametric Study}

The volume fraction profiles in FGMs are usually approximated by simple functions such as exponentials, powerlaws, and sigmoidal functions in addition to linear distributions. The use of a parametric form to represent the volume fraction profiles is fundamental to establish the right quantities of each phase at any given point along the axis where the properties vary. However, those functions must reflect the actual distribution of inclusions along the wall thickness, and in many cases simple functions are not able to represent well the measured data. The parametric study was based 
upon the volume fraction profiles obtained from Ulukoy et al. (2016) and shown in Figure 5. The material properties are given in Table 1.

Table 1: Material Properties

\begin{tabular}{cccc}
\hline Phase & E [GPa] & v \\
\hline Aluminum & 70.0 & 0.34 & 0.16 \\
SiC & 415.0 & & \\
\hline
\end{tabular}

The measured volume fraction of $\mathrm{SiC}$ is reported along the thickness of the wall of centrifugally cast cylindrical specimens. The experimental data was fitted to an exponential curve and to a cubic B-Spline function. This was so to quantify the divergences when assuming simpler curves (exponential) comparatively to curves that represent better the jaggedness of the measured data (B-Splines). The UMATs corresponding to each homogenization scheme were used for calculation of the hoop and radial stresses along the wall thickness.

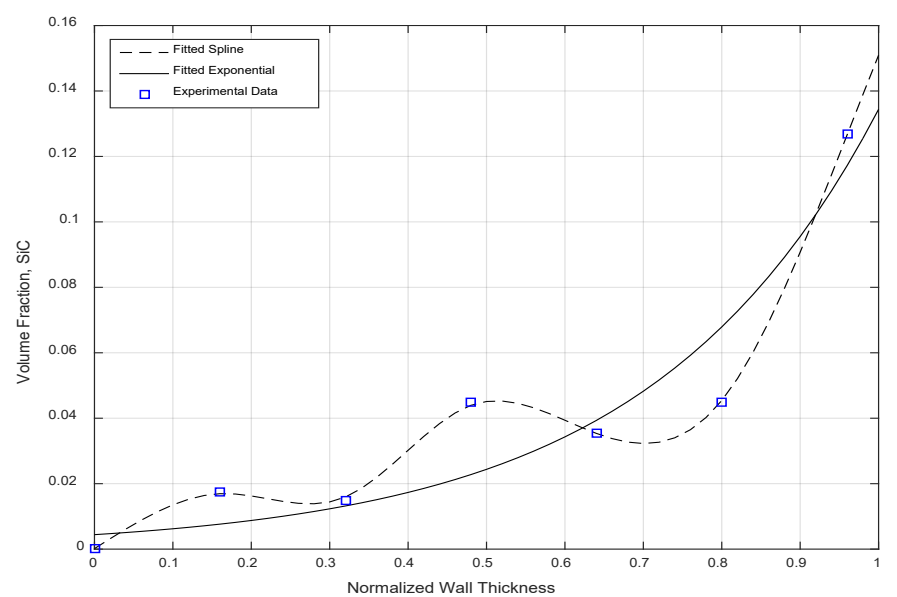

Figure 5: Fitted curve versus experimental volume fraction profile (Data from Ulukoy et al., 2016)

\section{RESULTS}

Figure 6 presents the calculated elastic modulus along the wall thickness for the volume fraction profile adopted in this work. It is clear that the waviness captured by the B-Spline curves reflects itself on the homogenized modulus. Another important point is the similarity between the results from the Mori-Tanaka and Generalized Self-Consistent methods, which is expected due to the low concentration of SiC as depicted in Figure 5. This fact carried on into the calculated radial and hoop stresses as seen in Figure 7 and 8 . The Poisson's ratio was considered to vary according to the volume fraction and was calculated using Equation (34).

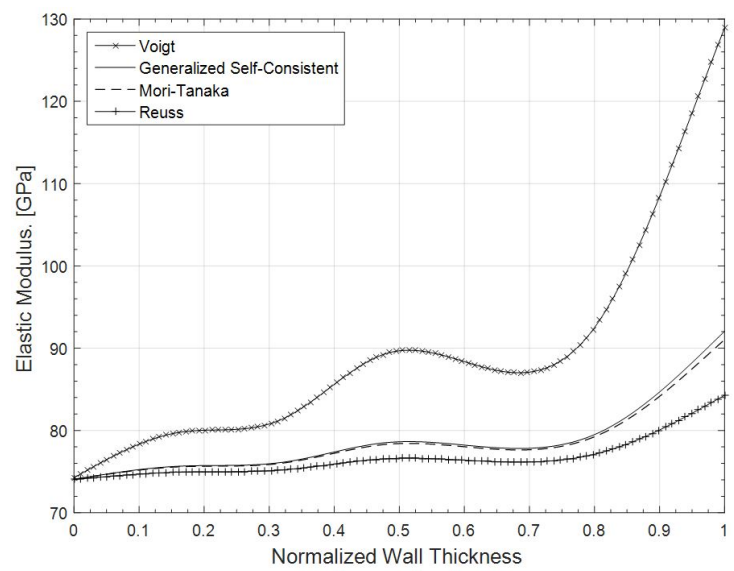

(a) B-Spline Fit

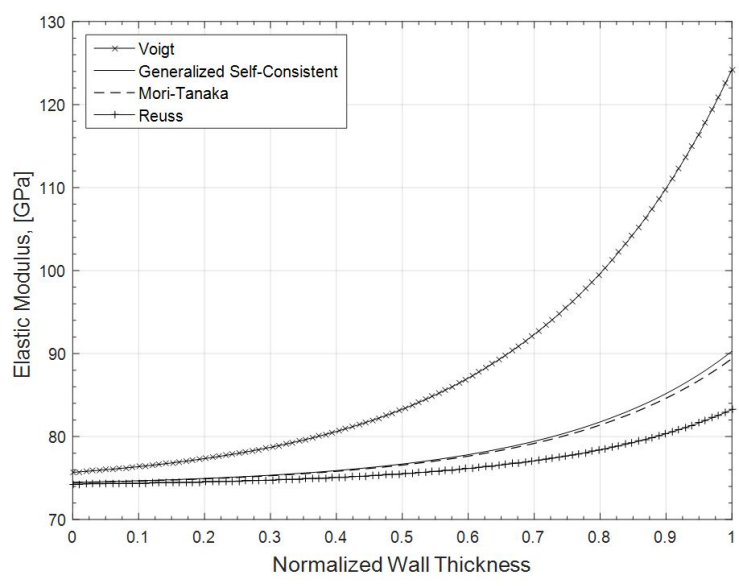

(b) Exponential Fit

Figure 6: Calculated elastic modulus with different fitted curves type 
In regards with radial stress, the overall trends were very close except for the Voigt model. The results from MoriTanaka and Generalized Self-Consistent are nearly indistinguishable for both B-Spline and exponential functions.

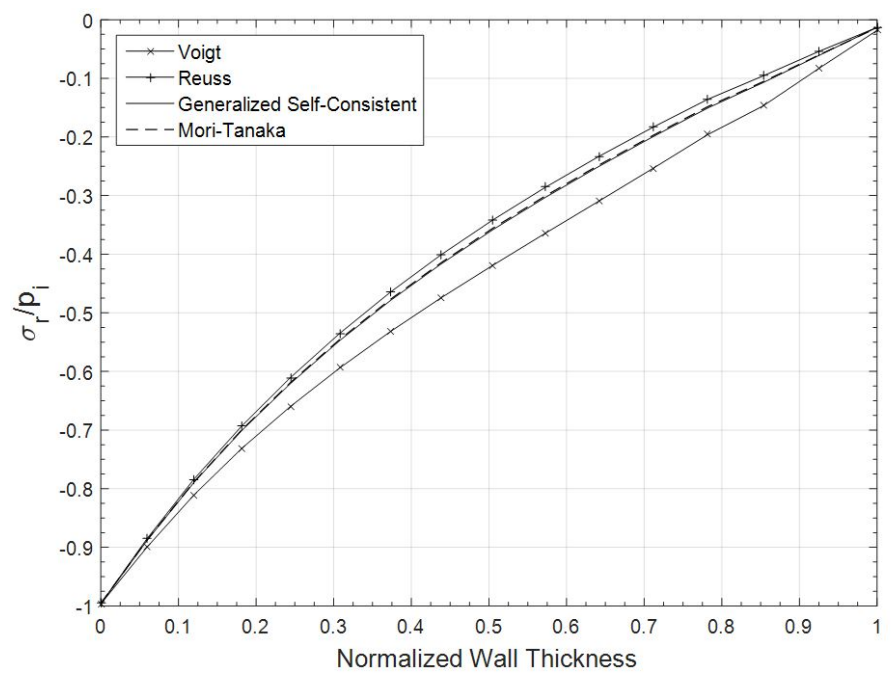

(a) B-Spline Fit

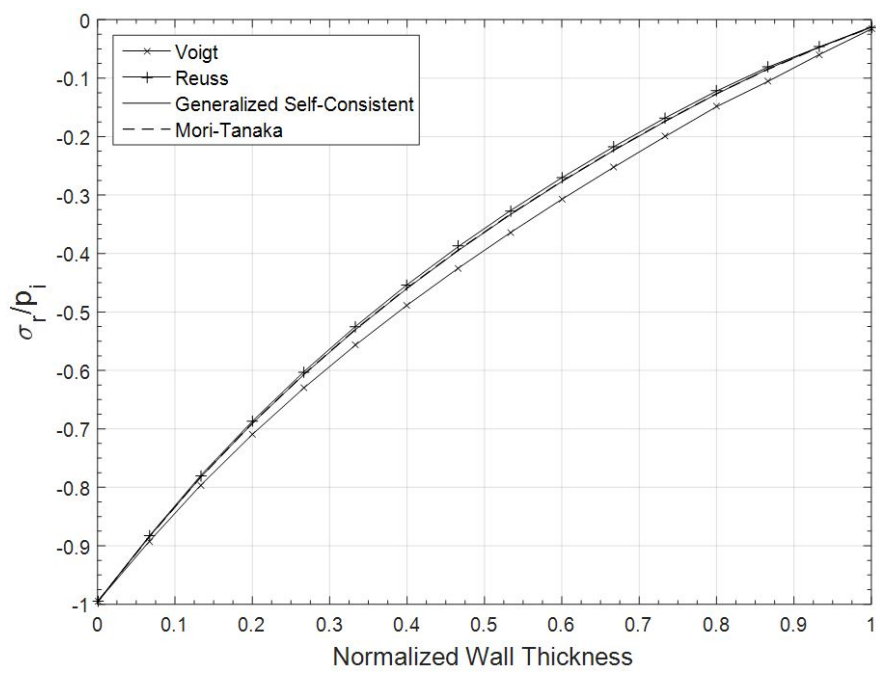

(b) Exponential Fit

Figure 7: Radial stress profiles from FE analysis

The hoop stress profiles presented a higher sensitivity to the type of homogenization scheme as well as the representation of the volume fraction profile. As seen in Figure 8a, the Voigt model showed an opposite trend for the variation of hoop stresses along the wall thickness. The higher value is seen on the outer face of the pipe while all other models show an opposite trend. Once again, the results from Mori-Tanaka and Generalized Self-Consistent are virtually the same.

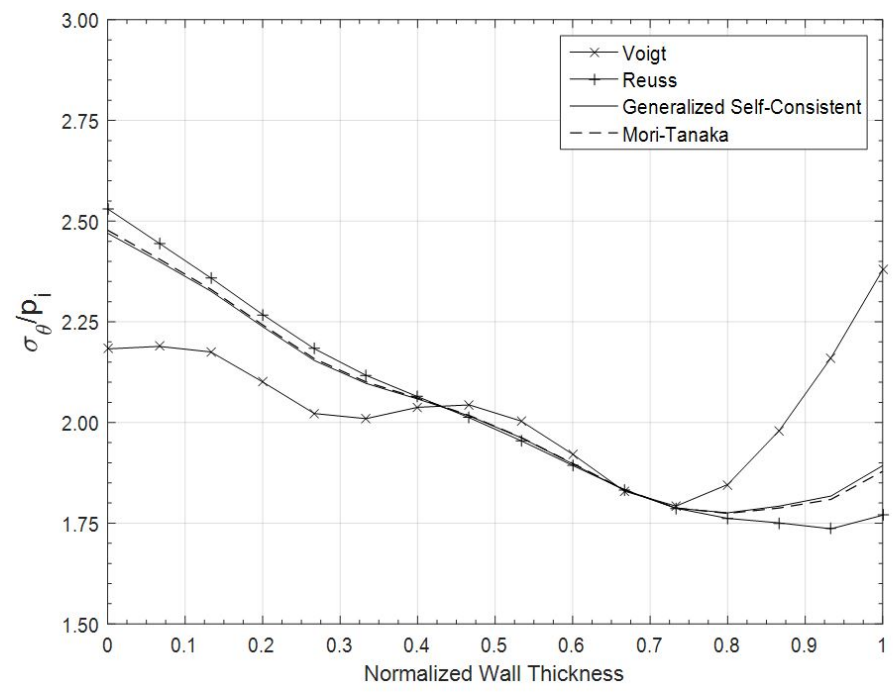

(a) B-Spline Fit

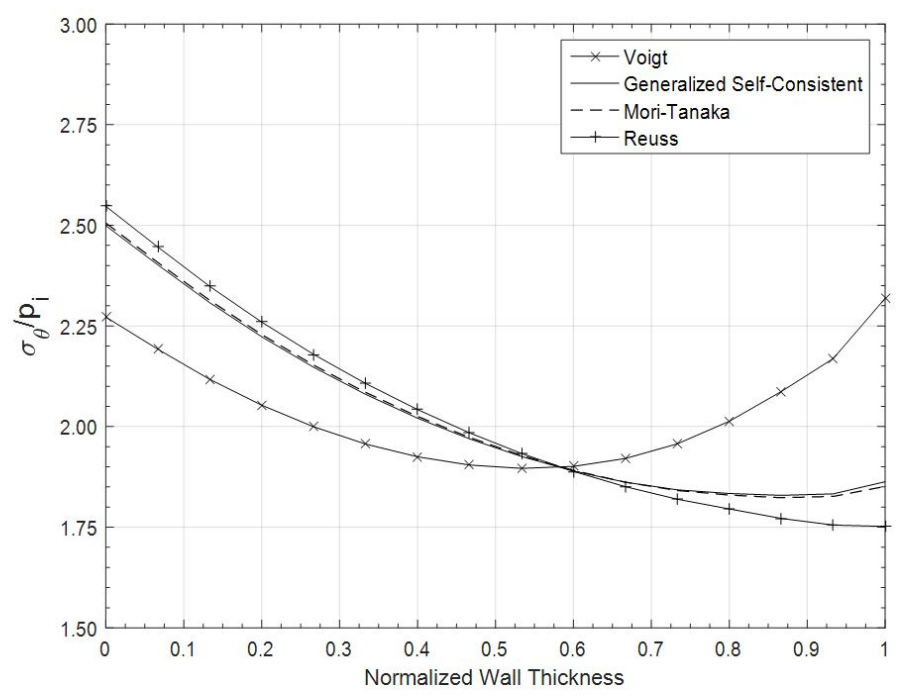

(b) Exponential Fit

Figure 8: Hoop stress profiles from FE analysis

Figure 9a compares the effect of different homogenization schemes. Figure $9 \mathrm{~b}$ shows the impact of considering the actual volume fraction profile with its inherent fluctuations (B-Splines) versus assuming a smoother approximation (exponential form). As can be seen, the homogenization scheme plays a major role on the final results of hoop stresses. 


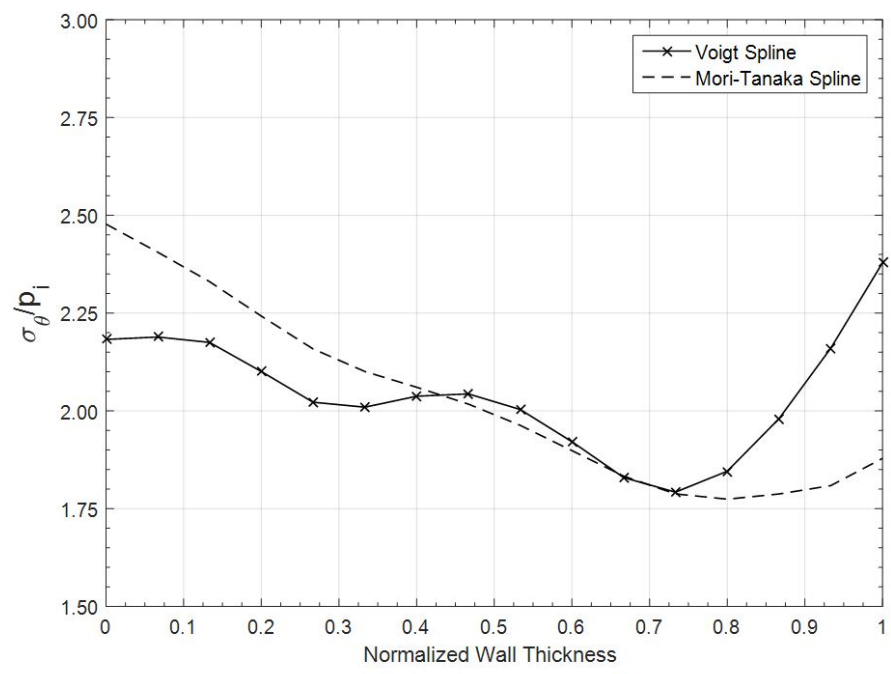

(a) Comparison between two different homogenization schemes

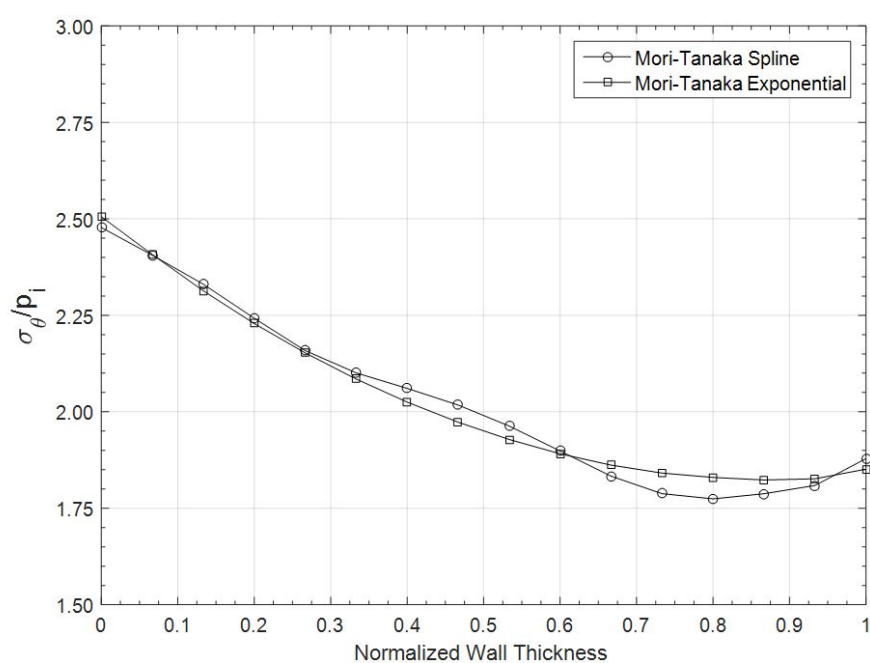

(b) Comparison between two different volume fraction profile approximation

Figure 9: Comparison between the hoop stress profiles for different scenarios

\section{CONCLUSIONS}

This work was aimed at investigating the influences of different forms of volume fraction description as well as the impact of using different homogenization schemes on the overall behavior of a FGM pressurized hollow cylinders. To achieve this goal, a set of user-material subroutines were developed and implemented into Abaqus. They were used to simulate an FGM pressurized pipe, where the volume fraction of the SiC particulate was accurately measured. The results obtained showed the importance of correctly describing the distribution of the particulate reinforcement (volume fraction of inclusions) along the wall thickness.

Regarding the different homogenization schemes, the values provided by the Mori-Tanaka and Generalized SelfConsistent yielded very close results. These two models also showed a very good agreement with experimental data. In spite of its large popularity, the Voigt model must be avoided due to its large discrepancies comparatively to the experimental results.

The assumption of an exponential variation may lead to unrealistic hoop stress distributions along the radial profile of the structural element. It may also mask potential issues related to stress concentration in some areas were the volume fraction concentration does not varies as smoothly as predicted by simple functions. The waviness of the volume fraction distribution also affects directly the calculated stress when the actual volume fraction profile is taken into account. This is an important fact to be considered when employing an FGM material because the strength of the material also varies with the volume of the reinforcing inclusions.

\section{ACKNOWLEDGEMENTS}

The financial support provided by CNPq (Conselho Nacional de Desenvolvimento Científico e Tecnológico) is gratefully acknowledged.

\section{References}

Akbarzadeh, A.H., A. Abedini, and Z.T. Chen. 2015. "Effect of Micromechanical Models on Structural Responses of Functionally Graded Plates." Composite Structures 119 (January): 598-609. doi:10.1016/j.compstruct.2014.09.031.

Bains, Preetkanwal Singh, Sarabjeet Singh Sidhu, and H. S. Payal. 2016. "Fabrication and Machining of Metal Matrix Composites: A Review." Materials and Manufacturing Processes 31 (5): 553-73. doi:10.1080/10426914.2015.1025976.

Benveniste, Y. 1987. "A New Approach to the Application of Mori-Tanaka's Theory in Composite Materials." Mechanics of Materials 6 (2): 147-57. doi:10.1016/0167-6636(87)90005-6. 
- - - 2008. "Revisiting the Generalized Self-Consistent Scheme in Composites: Clarification of Some Aspects and a New Formulation." Journal of the Mechanics and Physics of Solids 56 (10): 2984-3002. doi:10.1016/j.jmps.2008.06.006.

Chou, Tsu-Wei, Seiichi Nomura, and Minoru Taya. 1980. "A Self-Consistent Approach to the Elastic Stiffness of Short-Fiber Composites." Journal of Composite Materials 14 (3): 178-88. doi:10.1177/002199838001400301.

Christensen, R.M., and K.H. Lo. 1979. "Solutions for Effective Shear Properties in Three Phase Sphere and Cylinder Models." Journal of the Mechanics and Physics of Solids 27 (4): 315-30. doi:10.1016/0022-5096(79)90032-2.

Christensen, Richard M. 1990. "A Critical Evaluation for a Class of Micro-Mechanics Models." Journal of the Mechanics and Physics of Solids 38 (3): 379-404. doi:10.1016/0022-5096(90)90005-0.

Eshelby, J. D. 1957. "The Determination of the Elastic Field of an Ellipsoidal Inclusion, and Related Problems." Proceedings of the Royal Society A: Mathematical, Physical and Engineering Sciences 241 (1226): 376-96. doi:10.1098/rspa.1957.0133.

Gross, Dietmar, and Thomas Seelig. 2011. Fracture Mechanics. Mechanical Engineering Series. Berlin, Heidelberg: Springer Berlin Heidelberg. doi:10.1007/978-3-642-19240-1.

Hashin, Z. 1963. "Theory of Mechanical Behavior of Heterogeneous Media, Technical Report No 3." Philadelphia.

Hill, R. 1964. "Theory of Mechanical Properties of Fibre-Strengthened Materials: I. Elastic Behaviour." Journal of the Mechanics and Physics of Solids 12 (4): 199-212. doi:10.1016/0022-5096(64)90019-5.

1965. "A Self-Consistent Mechanics of Composite Materials." Journal of the Mechanics and Physics of Solids 13 (4): 213-22. doi:10.1016/0022-5096(65)90010-4.

Kainer, Karl U. 2006. Metal Matrix Composites: Custom-Made Materials for Automotive and Aerospace Engineering. 1st Editio. Verlag, Wiley-VCH.

Koizumi, M. 1997. “FGM Activities in Japan." Composites Part B: Engineering 28 (1-2): 1-4. doi:10.1016/S13598368(96)00016-9.

Li, Xian-Fang, and Xu-Long Peng. 2009. "A Pressurized Functionally Graded Hollow Cylinder with Arbitrarily Varying Material Properties." Journal of Elasticity 96 (1): 81-95. doi:10.1007/s10659-009-9199-z.

Liaw, P.K., R.E. Shannon, W.G. Clark, W.C. Harrigan, H. Jeong, and D.K. Hsu. 1995. "Nondestructive Characterization of Material Properties of Metal-Matrix Composites." Materials Chemistry and Physics 39 (3): 220-28. doi:10.1016/0254-0584(94)01431-F.

“MATWEB." 2018. www.matweb.com.

Mori, T, and K Tanaka. 1973. "Average Stress in Matrix and Average Elastic Energy of Materials with Misfitting Inclusions." Acta Metallurgica 21 (5): 571-74. doi:10.1016/0001-6160(73)90064-3.

Mura, Toshio. 1982. Micromechanics of Defects in Solids. Dordrecht: Springer Netherlands. doi:10.1007/978-94-011-9306-1.

Perdahcıoğlu, E. Semih, and Hubert J. M. Geijselaers. 2011. "Constitutive Modeling of Two Phase Materials Using the Mean Field Method for Homogenization." International Journal of Material Forming 4 (2): 93-102. doi:10.1007/s12289-010-1007-6.

Premkumar, M. K., W. H. Hunt, and R. R. Sawtell. 1992. "Aluminum Composite Materials for Multichip Modules." JOM 44 (7): 24-28. doi:10.1007/BF03222271.

Qu, SG, HS Lou, and XQ Li. 2016. "Influence of Particle Size Distribution on Properties of SiC Particles Reinforced Aluminum Matrix Composites with High SiC Particle Content." Journal of Composite Materials 50 (8): 1049-58. doi:10.1177/0021998315586864.

Reuss, A. 1929. "Berechnung Der Fließgrenze von Mischkristallen Auf Grund Der Plastizitätsbedingung Für Einkristalle ." ZAMM - Zeitschrift Für Angewandte Mathematik Und Mechanik 9 (1): 49-58. doi:10.1002/zamm.19290090104.

Santhosh Kumar, S, V Seshu Bai, K V Rajkumar, G K Sharma, T Jayakumar, and T Rajasekharan. 2009. "Elastic Modulus of Al$\mathrm{Si} /$ SiC Metal Matrix Composites as a Function of Volume Fraction." Journal of Physics D: Applied Physics 42 (17): 175504. doi:10.1088/0022-3727/42/17/175504.

Shen, Hui-Shen, and Zhen-Xin Wang. 2012. "Assessment of Voigt and Mori-Tanaka Models for Vibration Analysis of Functionally Graded Plates.” Composite Structures 94 (7): 2197-2208. doi:10.1016/j.compstruct.2012.02.018.

Sherif El-Eskandarany, M. 1998. "Mechanical Solid State Mixing for Synthesizing of SiCp/Al Nanocomposites." Journal of Alloys and Compounds 279 (2): 263-71. doi:10.1016/S0925-8388(98)00658-6. 
Stefanescu, Doru Michael. 2009. Science and Engineering of Casting Solidification, Second Edition. Boston, MA: Springer US. doi:10.1007/b135947.

Tucker III, Charles L., and Erwin Liang. 1999. "Stiffness Predictions for Unidirectional Short-Fiber Composites: Review and Evaluation." Composites Science and Technology 59 (5): 655-71. doi:10.1016/S0266-3538(98)00120-1.

Ulukoy, A, M Topcu, and S Tasgetiren. 2016. "Experimental Investigation of Aluminum Matrix Functionally Graded Material: Microstructural and Hardness Analyses, Fretting, Fatigue, and Mechanical Properties." Proceedings of the Institution of Mechanical Engineers, Part J: Journal of Engineering Tribology 230 (2): 143-55. doi:10.1177/1350650115594405.

Upadhyay, Anupama, and Ramvir Singh. 2012. "Prediction of Effective Elastic Modulus of Biphasic Composite Materials." Modern Mechanical Engineering 02 (01): 6-13. doi:10.4236/mme.2012.21002.

Vignoli, Lucas Lisbôa, and Marcelo Amorim Savi. 2018. "Multiscale Failure Analysis of Cylindrical Composite Pressure Vessel: A Parametric Study." Latin American Journal of Solids and Structures 15 (11). doi:10.1590/1679-78254323.

Voigt, W. 1889. "Ueber Die Beziehung Zwischen Den Beiden Elasticitätsconstanten Isotroper Körper." Annalen Der Physik 274 (12): 573-87. doi:10.1002/andp.18892741206. 